\title{
On the Taylor hypothesis in forced unsteady wall flows
}

\begin{abstract}
Experiments on the modulation characteristics of the wall shear stress $\tau^{\prime}$-longitudinal velocity $u^{\prime}$ and $u^{\prime}-u^{\prime}$ space-time correlations are reported in a forced turbulent channel flow in a wide range of imposed frequencies. The resulting integral and Taylor scale properties are discussed in detail in the low buffer layer under steady and unsteady flow conditions. It is shown that the small-scale turbulence is sensitive to the imposed unsteadiness since the amplitude and phase of the Taylor length scale vary considerably in the imposed frequency range investigated here. The Taylor hypothesis is acceptably valid in steady and unsteady wall layers just above the low buffer layer. Production and instantaneous pressure gradients are mostly responsible for the deviation of the frozen turbulence-state in the viscous and low buffer sublayers.
\end{abstract}

\section{Introduction}

Unsteady turbulent wall layers are non-canonical complex flows. The complexity is due on one hand, to the introduction of the additional parameters induced by the unsteadiness such as the amplitude and frequency of the imposed velocity oscillations. That renders the parametrical study difficult. On the other hand, unsteadiness implies equivalently the forcing of the near wall turbulence whose response cannot be easily determined because of its inherent non-linearity. This is the reason why the modeling of the forced internal flows is still a challenge despite the considerable efforts made in the last decade (Tardu and Da Costa 2004 and the references within).

S. Tardu $(\bowtie) \cdot$ P. Vezin

Laboratoire des Ecoulements Géophysiques et Industriels (LEGI), CNRS-INPG-UJF, B.P. 53 X, 38041 Grenoble Cédex, France

E-mail: sedat.tardu@hmg.inpg.fr
Internal turbulent flows subject to forced sinusoidal velocity oscillations are encountered in several practical applications such as in helicopter blade aerodynamics, in turbomachinery, aeroacoustics, in internal combustion engines, in animal propulsion and bio-fluid dynamics. Intensive past research (Tu and Ramaprian 1983; Mao and Hanratty 1986; Finnicum and Hanratty 1998; Brereton et al. 1990; Tardu and Binder 1993; Tardu et al. 1994) turned essentially on the parametrical studies of the flow field. There are three main characteristics of the forced wall flows on which consensus is well established by now. First of all the time mean flow is unaffected by the forcing even at high imposed amplitudes and frequencies. There is a coexistence of a viscous Stokes type oscillating longitudinal velocity field with a time mean turbulent flow when the imposed period is comparable with the median time-scale of the wall turbulence. The turbulence in this regime cannot follow the rapid temporal variation of the shear and becomes frozen during the oscillation cycle. Only a few studies exist in the literature on the response of the fine structure of the turbulence in unsteady flows (Tardu and Binder 1997) and of the length scales concerning the spanwise structure of the flow (Finnicum and Hanratty 1998; Brereton and Hwang 1994; Tardu and Vezin 2004). An interesting quantity is the Taylor scale, which is directly related to the average dimensions of the eddies responsible for dissipation. The reaction of this quantity to the imposed unsteadiness would reflect therefore the reaction of small-scale turbulence. One immediate question arises: Is the Taylor hypothesis valid in unsteady wall layers? The answer is not immediate since the reaction of turbulence may have curious facets, especially, when the imposed frequency is high.

Experimentalists to evaluate the longitudinal derivatives of the fluctuating velocity field from temporal series commonly use the Taylor hypothesis. Despite the fact that it is widely used and the existence of large literature on the subject, there is still no clear assessment where exactly the frozen turbulence hypothesis can be used without warning in the wall bounded shear flows. The 
earliest investigations of Lin (1953) and Sternberg (1967) suggested that the large eddies induce large deviations from the frozen state because of the distorting effect of the mean shear on the turbulent vorticity field. This invalidates the Taylor hypothesis in a large part of the boundary layer. Yet, both the large eddy and direct numerical simulations based research (Piomelli et al. 1989; Kim and Hussain 1992) indicated more recently that time and streamwise derivatives are well correlated immediately above the low buffer layer. The mechanisms that deviate the convection velocity from the local velocity in the low buffer and viscous sublayers are still unclear.

To our knowledge, there is no published data on the longitudinal length scales and convection velocity modulation characteristics in forced turbulent internal flows. That might be important in, for example, unsteady aeroacoustic applications to determine the propagation velocity of the source. Experiments on the modulation characteristics of the wall shear stress $\tau^{\prime}$-longitudinal velocity $u^{\prime}$ and $u^{\prime}-u^{\prime}$ space-time correlations are reported here in a wide range of imposed frequencies. The resulting integral and Taylor scales properties are discussed in detail in the low buffer layer under steady and unsteady flow conditions. A discussion based on the direct numerical simulation data in a low Reynolds number canonical turbulent channel flow is conducted in somewhat a different way from the previous investigations to determine the basic mechanisms that validate or invalidate the Taylor hypothesis near the wall.

\section{Experimental set up and data reduction}

The experiments were conducted in the unsteady water channel described in detail in Tardu et al. (1994). A specific pulsating device generates the imposed sinusoidal velocity oscillations. The mean, amplitude and frequency of the velocity modulation at the centerline can be varied independently. High imposed amplitudes and frequencies can be applied this way.

The centerline velocity was held constant at $\bar{U}_{\mathrm{c}}=17.5 \mathrm{~cm} / \mathrm{s}$. This corresponds to a friction velocity of $\bar{u}_{\tau}=0.85 \mathrm{~cm} / \mathrm{s}$ and a Reynolds number based on the half height $h$ of the channel of $R e=\bar{U}_{\mathrm{c}} h / v=8,800$ where $v$ is the kinematic viscosity. The imposed amplitude was $20 \%$ of the centerline velocity throughout the whole study. The imposed frequency in wall units $f^{+}=f\left(v / \bar{u}_{\tau}^{2}\right)$ varied by a factor 24 from $f^{+}=2.2 * 10^{-4}$ to $f^{+}=60^{*} 10^{-4}$. Hereafter ()$^{+}$designates variables normalized by $v$ and $\bar{u}_{\tau}$. In terms of the frequency parameter $l_{s}^{+}=\sqrt{1 /\left(\pi f^{+}\right)}$which is the viscous Stokes length normalized by the inner length scale $l_{v}=v / \bar{u}_{\tau}$ and which was previously used by Tardu et al. (1994) the imposed frequency range investigated in this study covers $l^{+}{ }_{s}=38-8$.

The streamwise space-time correlations of both the fluctuating wall shear stress $\tau^{\prime}$ and longitudinal velocity fluctuations $u^{\prime}$, together with $u^{\prime}-u^{\prime}$ correlations were performed by means of a flush-mounted TSI-1268 W hot film at the wall and two TSI 1276-10W hot films in the flow. The probe displacements were done by use of controlled traversing mechanisms. The sensitive parts of the hot films are $8 l_{v}$ for the wall probe and $4 l_{v}$ for the hot films. The latter were operated at constant temperature with 5-8\% overheat by AHARONI AN-1003 anemometer units. These low overheats allowed the avoidance of the thermal wake effects. The calibration of the hot films has been done in situ as described by Tardu and Binder (1993) and Tardu et al. (1994). Due to the long record length, the calibration was checked for the temperature drift and repeated before and after each measurement.

The analog to digital conversion was achieved with an Analog-Device RTI-800 board (accuracy 11 bit + sign; 8 channel) installed in a PC computer. The sampling frequency was $1.5\left(\bar{u}_{\tau}^{2} / v\right)$. The signals were filtered with accurate cut-off frequencies. The minimum record length was $T_{\mathrm{r}}=10,500\left(h / \bar{U}_{c}\right)=218.10^{3}\left(v / \bar{u}_{\tau}^{2}\right)$ for each signal representing $50 \mathrm{~min}$ for each experiment. The duration of the data here is about 15 times longer than in steady flow since in unsteady flow convergence has to be achieved in each bin used in the phase average.

The classical triple decomposition is used. A quantity $q$ is decomposed into a mean $\bar{q}$ an oscillating $\tilde{q}$ and fluctuating $q^{\prime}$ component. The angle brackets designate the phase average i.e. $\langle q\rangle=\bar{q}+\tilde{q}$. The modulation characteristics of $\langle q\rangle$ are described by the amplitude $A_{\tilde{q}}$ and phase $\phi_{\tilde{q}}$ of the fundamental mode $\tilde{q}=A_{\tilde{q}} \cos \left(2 \pi f t+\phi_{\tilde{q}}\right)$. The relative amplitude $a_{\tilde{q}}=A_{\tilde{q}} / \bar{q}$ is also introduced for convenience. To test for the existence of subharmonics phase averaging over six imposed periods have been performed and the amplitudes of the harmonics have been analyzed through a discrete Fourier analysis as part of the usual procedure. The amplitudes of the first fifth harmonics are roughly eight times smaller than the sixth one. The highest imposed frequency investigated here which is 100 times smaller than the Kolmogoroff frequency, and roughly 20 times smaller than the Taylor frequency is not high enough to generate (eventual) subharmonics.

The phase averaged $\tau^{\prime}-u^{\prime}$ and $u^{\prime}-u^{\prime}$ correlation coefficients are defined as:

$$
\begin{aligned}
& \left\langle R_{\tau^{\prime} u^{\prime}}\left(x^{+}, y^{+}, t^{+}\right)\right\rangle \\
& =\frac{\left\langle\tau^{\prime}\left(x^{+}=0, z^{+}=0, t^{+}\right) u^{\prime}\left(x^{+}, y^{+}, z^{+}=0, t^{+}\right)\right\rangle}{\sqrt{\left\langle\tau^{\prime} \tau^{\prime}\right\rangle\left\langle u^{\prime} u^{\prime}\right\rangle}}
\end{aligned}
$$

and

$$
\begin{aligned}
& \left\langle R_{u^{\prime} u^{\prime}}\left(x^{+}, y^{+}, t^{+}\right)\right\rangle \\
& =\frac{\left\langle u^{\prime}\left(x^{+}=0, y^{+}, z^{+}=0, t^{+}\right) u^{\prime}\left(x^{+}, y^{+}, z^{+}=0, t^{+}\right)\right\rangle}{\left\langle u^{\prime} u^{\prime}\right\rangle}
\end{aligned}
$$

where, $x, y, z$ are respectively, the streamwise, wall normal and spanwise coordinates. They were measured for eight imposed frequencies and 20 different $x$ positions. Both the long record length and high sampling frequency resulted in huge amount of data of several GB. The smallest streamwise offset in the $\left\langle R_{\text {utul }}\right\rangle$ measurements 
were 12 to avoid thermal wake effects. The main aim of this study is to determine whether the Taylor hypothesis is valid or not in the buffer layer and in the presence of the oscillating shear, since the log-layer and the plug flow zone do not present great interest for this aspect. The oscillating shear layer thickness decreases as the frequency increases in the unsteady wall layers (Tardu et al. 1994). And it is constrained into $y^{+}<16$ at the highest frequency investigated here. Thus the emphasis here is on the measurements performed at $y^{+}=12$. A limited number of experiments have also been conducted at $y^{+}=30$ and $y^{+}=50$. They are only briefly mentioned.

\section{Results}

\subsection{Summary of flow characteristics}

Past research has shown that there is a coexistence of a purely viscous oscillating Stokes component with an unaltered time mean base flow when the imposed fre- quency is larger than $f^{+}=0.003$ (see e.g., Tardu et al. 1994). Figure 1a shows the modulation characteristics of the wall shear stress $\langle\tau\rangle$ in terms of the Stokes length $l_{s}^{+}=\sqrt{1 /\left(\pi f^{+}\right)}$. It is seen that when $l^{+}{ }_{s}<10$, the amplitude and phase of $\langle\tau\rangle$ coincides well with $A_{\tilde{\tau} \text { Stokes }}=\sqrt{2} \rho v\left(A_{\tilde{u} c} / l_{s}\right)$ and $\phi_{\tilde{\tau} \text { Stokes }}-\phi_{\tilde{u} c}=45$. Thus, under the present conditions with $a_{\tilde{u} c}=0.20$ at the centerline, the shear in the viscous sublayer has modulation amplitudes that vary between $a_{\tilde{\tau}}=0.40$ in the low frequency, quasi-steady regime, to as large as $a_{\tilde{\tau}}=0.60$ at the highest imposed frequency investigated here. These values show how the forcing of the wall turbulence is important in this investigation.

Figure 1b shows the relative amplitude of the wall shear stress intensity $\left\langle\tau^{\prime} \tau^{\prime}\right\rangle$ related to the relative amplitude of the wall shear stress $\langle\tau\rangle$ versus the imposed frequency $f^{+}$. The corresponding ratio $a_{u^{\prime} \tilde{u}^{\prime}} / a_{\tilde{u}}$ of the longitudinal turbulent intensity modulation and that of the local velocity $\langle u\rangle$ at $y^{+}=12$ is also shown in Fig. 1b. Note first that the measurements in the low frequency regime agree well with the expected quasi-
Fig. 1 Flow characteristics. Modulation amplitude and phase of the wall shear stress (a), modulation amplitude of the turbulent wall shear stress intensity and of the longitudinal turbulent intensity (b) versus the imposed frequency. The imposed amplitude at the centerline is $20 \%$ of the mean velocity
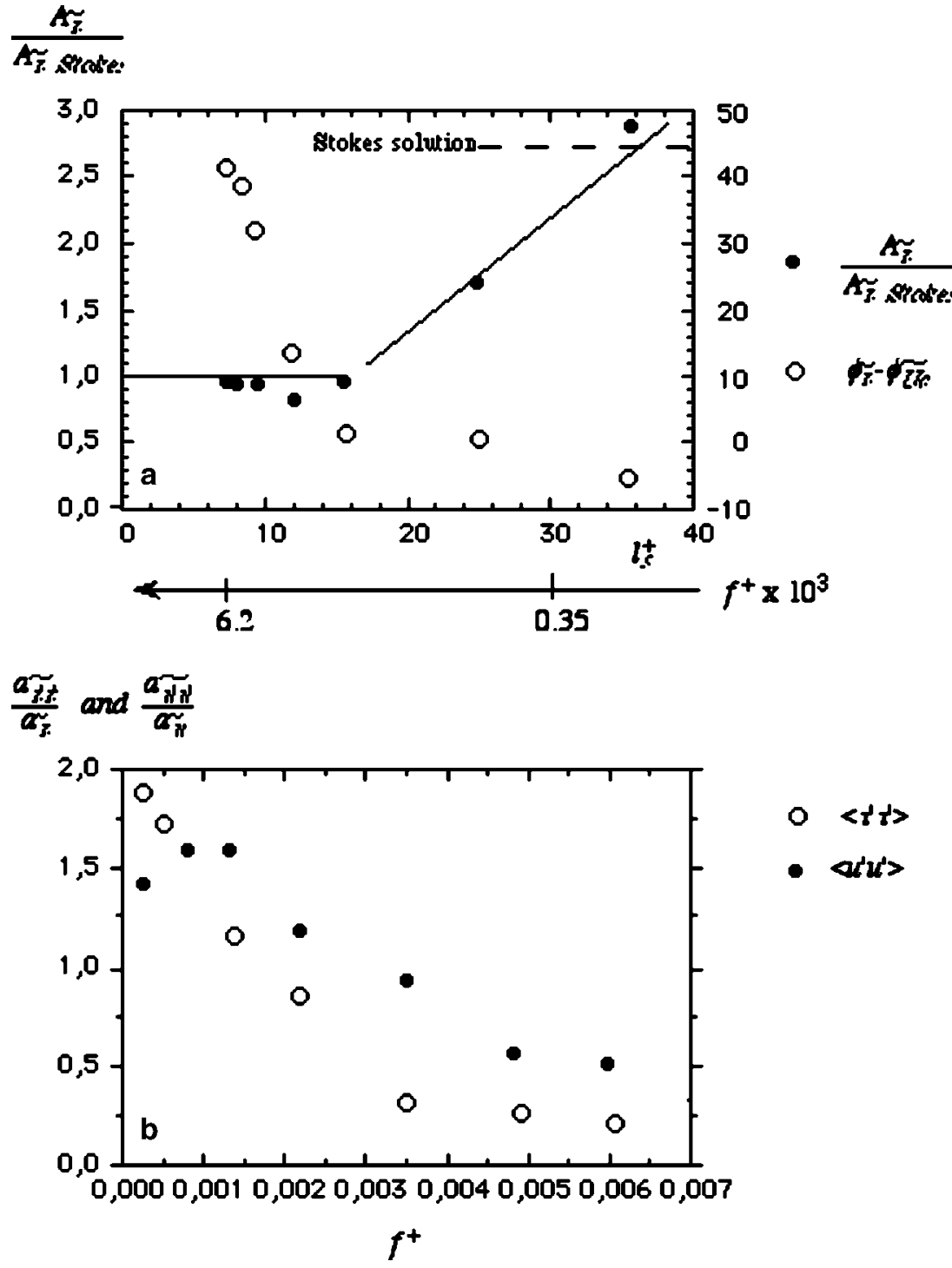
steady behaviors. In the low frequency range, one has $\left\langle\tau^{\prime} \tau^{\prime}>_{q s} /<\tau>_{q s}^{2}=0.38^{2}\right.$ and a Taylor series analysis of this relationship leads to $a_{\tau^{\prime} \tau^{\prime} q s}=2 a_{\tilde{\tau} q s}=4 a_{u \tilde{c}}$ reaching values as high as 0.8 under the present experimental conditions. The modulations of both $\left\langle\tau^{\prime} \tau^{\prime}>\right.$ and $<u^{\prime}$ $u^{\prime}>$ decrease when the imposed frequency increases but the decay in $a_{\tau^{\prime} \tau^{\prime}} / a_{\tilde{\tau}}$ is more pronounced and takes place earlier. These results are in good agreement with previously published data. The weakening of the modulation of the turbulence in this frequency regime may be explained by the fact that the near wall flow responds only partly to the rapid temporal changes of the shear. Both the experimental and modeling aspects concerning these particular points are beyond the scope of this paper and may be found in Tardu and Da Costa (2005).

\subsection{Modulation characteristics of the streamwise correlation coefficient}

The time mean correlation coefficient $\bar{R}_{\tau^{\prime} u^{\prime}}$ at $y^{+}=12$ between the fluctuating wall shear stress $\tau^{\prime}$ and streamwise velocity fluctuations $u^{\prime}$ is plotted versus the streamwise offset $x^{+}$in Fig. 2a for five imposed frequencies investigated so far. The excellent collapse of $\bar{R}_{\tau^{\prime} u^{\prime}}$ for different values of the frequency parameter $l^{+}{ }_{s}$ and good correspondence with the steady flow profile are reminiscent of the insensitivity of the time mean correlation coefficient to the imposed unsteadiness.

It is seen in Fig. 2a that the maximum of the time mean correlation coefficient $\bar{R}_{\tau^{\prime} u^{\prime}}$ is located at $x^{+} \approx 50$. This can be explained by the tilting of the hairpin-like vortical structures as they develop in the inner (Head and Bandyopadhyay 1981). The vertical growth of a hairpin vortex is governed by its induced velocity and shear effect, resulting in an angle of $45^{\circ}$ when both effects balance each other in the log layer. Several angles may be identified in a hairpin during its spatial growth. The flow visualizations of Falco (1977) and the timespace correlations performed by Brown and Thomas (1977) and more recently by Wark and Nagib (1991) suggest a structure angle of about $10^{\circ}$ in the low-buffer layer. The measurements presented in Fig. 2 a give, in
Fig. 2 Time mean correlation coefficient versus the streamwise offset in wall units for several imposed frequencies described by the parameter $l_{s}^{+}=\sqrt{1 /\left(\pi f^{+}\right)}$. a Wall shear stress-velocity fluctuations at $y^{+}=12$. b Streamwise velocity correlations at $y^{+}=12$

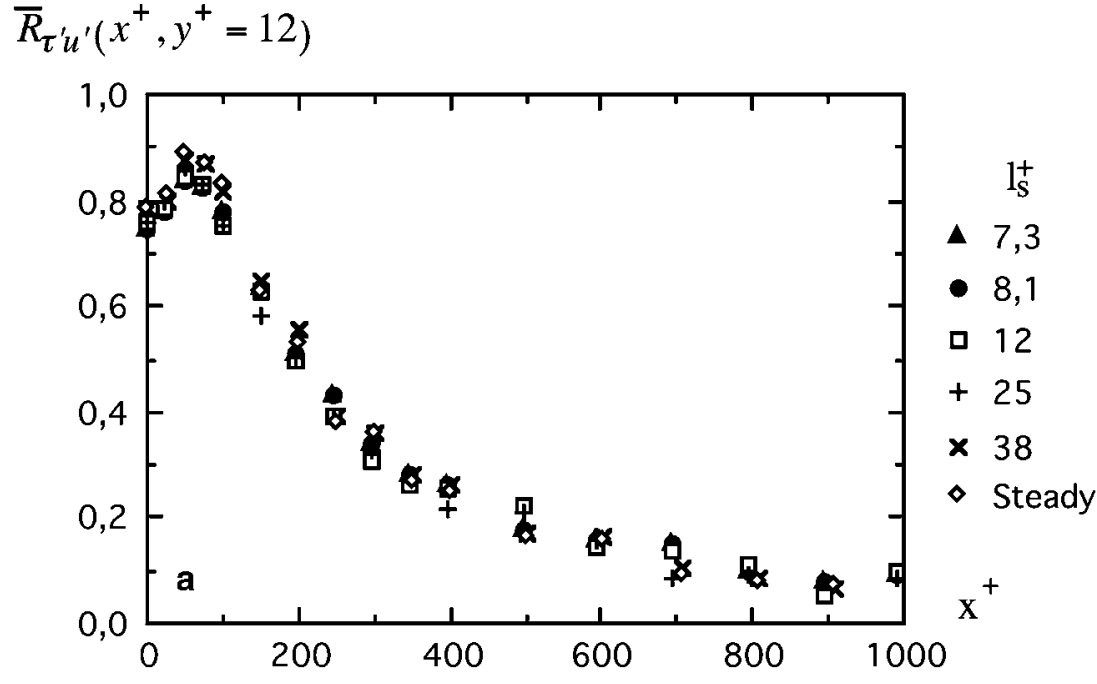

$1-\bar{R}_{u^{\prime} u^{\prime}}\left(x^{+}, y^{+}=12\right)$

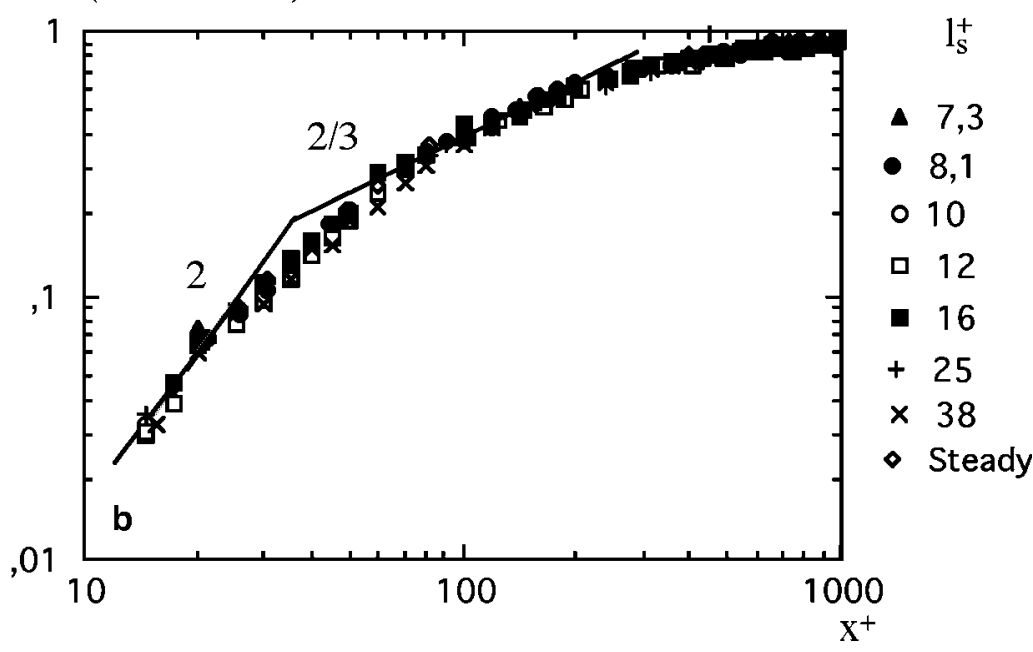


return, an angle of $\alpha=\tan ^{-1}\left(y^{+} / x^{+}\right) \approx 11$ when $x^{+}=50$ and $y^{+}=12$. This value is in good agreement with the previous results quoted.

Figure $2 \mathrm{~b}$ shows the time mean of the phase averaged fluctuating velocity correlation coefficient $\left\langle R_{u^{\prime} u^{\prime}}\right\rangle$. The $1-\bar{R}_{u^{\prime} u^{\prime}}$ profiles are presented versus $x^{+}$in logarithmic scales. They compare in a satisfactory way with the results of McLean (1991) obtained at $R e_{\theta}=1,500$ in a steady turbulent boundary layer. The correlation coefficient varies like $1-\bar{R}_{u^{\prime} u^{\prime}} \propto x^{2 / 3}$ in the inertial subrange wherein the one-dimensional power spectral density is $E(k) \propto k^{-5 / 3}$. The range of the streamwise offset for the inertial behavior is narrow near the wall than in the log layer, and the results of McLean suggest that $E(k) \propto k^{-5 / 3}$ for $70<x^{+}<200$ in the buffer layer. The trend of the data in Fig. 1b confirms these arguments. For small values of the offset $x^{+}$the mean correlation coefficient should vary like $1-\bar{R}_{u^{\prime} u^{\prime}} \propto x^{2}$, because of the relationship $\bar{R}_{u^{\prime} u^{\prime}}=1-\left(x^{2} / \bar{\lambda}_{T x}^{2}\right)$ near $x \approx 0$, relating $\bar{R}_{u^{\prime} u^{\prime}}$ to the streamwise Taylor scale $\bar{\lambda}_{T x}$. This is also well established for $x^{+}<30$ in Fig. 2b. Note finally that both $\bar{R}_{\tau^{\prime} u^{\prime}}$ and $\bar{R}_{u^{\prime} u^{\prime}}$ extend to values as large as $x^{+}=1,000$.
Detailed analysis of the amplitude and phase of the cyclic modulation of the correlation coefficients revealed that the modulation characteristics of $\left\langle R_{\tau^{\prime} u^{\prime}}\right\rangle$ and $\left\langle R_{u^{\prime} u^{\prime}}\right\rangle$ are qualitatively similar. Therefore, we will only discuss the amplitude $A_{\tilde{R} u^{\prime} u^{\prime}}$ and phase $\phi_{\tilde{R} u^{\prime} u^{\prime}}$ of the phaseaveraged correlation coefficient $\left\langle R_{u^{\prime} u^{\prime}}\right\rangle$ to be brief.

In Fig. 3a, the amplitude $A_{\tilde{R} u^{\prime} u^{\prime}}$ is scaled with $A_{\tilde{R} u^{\prime} u^{\prime} q s}$ which is the amplitude that $\left\langle R_{u^{\prime} u^{\prime}}\right\rangle$ would have in the quasi-steady low imposed frequency limit. Generally speaking, the correlation coefficient $\bar{R}_{u^{\prime} u^{\prime}}$ in the canonical steady turbulent wall layer depends upon the Reynolds number, the wall normal and streamwise offsets i.e., $\bar{R}_{u^{\prime} u^{\prime}}=F\left(R e, y^{+}, x^{+}\right)$. In the very low frequency range, and in a similar manner, one has $\left\langle R_{u^{\prime} u^{\prime} q s}\right\rangle=F\left(\langle R e\rangle,\left\langle y^{+}\right\rangle,\left\langle x^{+}\right\rangle\right)$where $F$ is the same function as in steady flow. In this relationship,
$\langle R e\rangle=\frac{h\left\langle U_{c}\right\rangle}{v}$,
$\left\langle y^{+}\right\rangle=y \frac{\left\langle u_{\tau}\right\rangle}{v}$
and $\left\langle x^{+}\right\rangle=x \frac{\left\langle u_{\tau}\right\rangle}{v}$.

If the imposed amplitude is small enough, a Taylor expansion gives to the first order:
Fig. 3 Amplitude (a) and phase (b) of the streamwise velocity correlations at $y^{+}=12$ versus the imposed frequency at different streamwise offsets
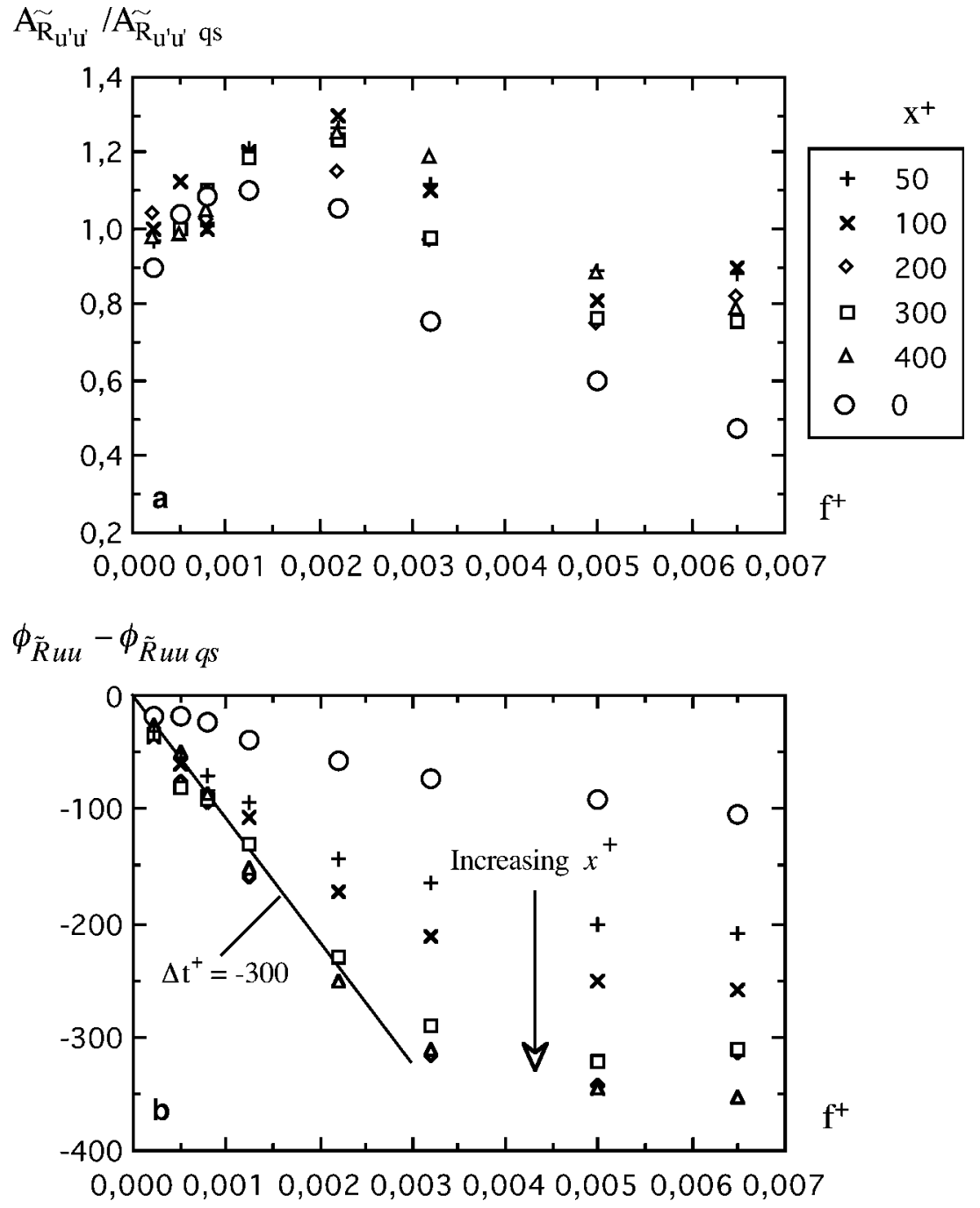
$A_{\tilde{R} u^{\prime} u^{\prime} q s} \approx\left[\frac{\partial F}{\partial x^{+}} x^{+}+\frac{\partial F}{\partial y^{+}} y^{+}+\frac{\partial F}{\partial R e} R e\right] a_{u \tilde{c}}$.

According to McLean (1991, p. 38) the effect of the Reynolds number on the inner scaled correlation coefficient is weak at $x^{+}<400$ in the buffer layer. Close inspection of his data shows also that $\bar{R}_{u^{\prime} u^{\prime}}$ varies only slightly with $y^{+}$close to the wall. Supposing therefore that $\left(\partial F / \partial x^{+}\right)>>\left(\partial F / \partial y^{+}\right)$and $\left(\partial F \partial x^{+}\right)>>(\partial F / \partial R e)$, and using a polynomial approximation $\bar{R}_{u^{\prime} u^{\prime}} \approx \sum_{i=1}^{n} \alpha_{i} x^{+i}$, one has $A_{\tilde{R} u^{\prime} u^{\prime} q s} \approx a_{u \tilde{c}} \sum_{i=1}^{n} i \alpha_{i} x^{+i}$. This approximation (with $n=5$ ) was confronted to the measurements taken at a particularly low imposed frequency $l^{+}{ }_{s}=60$. Good collapse between the computed and measured profiles was found.

The $A_{\tilde{R} u^{\prime} u^{\prime}} / A_{\tilde{R} u^{\prime} u^{\prime} q s}$ profiles versus the imposed frequency at different streamwise offsets are fairly well regrouped in Fig. 3a. They also compare qualitatively well with the amplitude of the streamwise intensity $A_{u^{\prime} u^{\prime}} / A_{u^{\prime} \tilde{u}^{\prime} q s}$ which is the asymptotic limit of $A_{\tilde{R} u^{\prime} u^{\prime}} / A_{\tilde{R} u^{\prime} u^{\prime} q s}$ at $x^{+}=0$. This shows that the unsteadiness is uniformly felt along the elongated quasi-streamwise structures. The ratio $A_{\tilde{R} u^{\prime} u^{\prime}} / A_{\tilde{R} u^{\prime} u^{\prime} q s}$ increases first from the quasi-steady limit and decreases subsequently in the relaxation regime $f^{+}>0.002$.

The phase shift of the correlation coefficient $\Delta \phi_{\tilde{R} u^{\prime} u^{\prime}}=\phi_{\tilde{R} u^{\prime} u^{\prime}}-\phi_{\tilde{R} u^{\prime} u^{\prime} q s}$ is shown in Fig. 3b. The time lag $\Delta t_{\tilde{R} u^{\prime} u^{\prime}}^{+}=\Delta \phi_{\tilde{R} u^{\prime} u^{\prime}}\left(l_{s}^{+2} / 2\right)$ is roughly constant and close to 300 wall units in the low imposed frequency range. For $f^{+}>0.001$, however, the phase shift increases with $x^{+}$. Large time lags mean high inertia. Thus, largescale structures are associated with high relaxation times, as expected.

\subsection{Integral scale}

The modulation characteristics of the streamwise integral scale $\left\langle\Lambda_{x}\right\rangle=\int_{0}^{\infty}\left\langle R_{u^{\prime} u^{\prime}}\right\rangle \mathrm{d} x$ at $y^{+}=12$ are recapitulated in Fig. 4. The time mean integral scale is $\bar{\Lambda}_{x}^{+}=280$ and it is unaffected by the imposed unsteadiness. This has to be compared with the streamwise length of the quasi-streamwise vortices in the buffer layer, that vary between 100 and 400 in wall units. The spanwise integral scale has also been measured under the same experimental conditions (Tardu and Vezin 2004) leading to $\bar{\Lambda}_{z}^{+}=24$ (not reported here). The ratio $\bar{\Lambda}_{x}^{+} / \bar{\Lambda}_{z}^{+} \approx 12$ is in good agreement with McLean (1991) who has shown that $\bar{\Lambda}_{x}^{+} / \bar{\Lambda}_{z}^{+}$is approximately constant in the inner layer, even though both scales depend on $\operatorname{Re}$ and $y^{+}$.

Figure 4a shows the relative amplitude of $\left\langle\Lambda_{x}\right\rangle$ scaled with $a_{u \tilde{c}}$ versus the imposed frequency. It turns out that the streamwise integral scale modulation amplitude does not depend upon $f^{+}$and that $a_{\Lambda \tilde{x}} \approx 0.4 a_{u \tilde{c}}$ with only a slight decrease at the highest frequency. The integral scale is modulated around its time mean value because it is a function of both the Reynolds number and the distance to the wall, in steady flow. Therefore, in the quasi-steady regime $\frac{\left\langle\Lambda_{x}\right\rangle}{h}=F\left(\langle R e\rangle,\left\langle y^{+}\right\rangle\right)$, and a simple analysis of the first order implies

$\frac{A_{\Lambda \tilde{x}}}{h} \approx\left[\frac{\partial F}{\partial \operatorname{Re}} \operatorname{Re}+\frac{\partial F}{\partial y^{+}} y^{+}\right] a_{u \tilde{c}}$.

A close inspection of the McLean's data shows that at sufficiently large $R e$ numbers $\bar{\Lambda}_{x} / h \propto \ln \left(y^{+}\right)$for $y^{+}<150$, and that $\bar{\Lambda}_{x}$ increases by a factor 2 when $y^{+}$ varies by a decade. Furthermore, the integral scale decreases slowly with $R e$, by about $40 \%$ for $20.10^{3}<R e<40.10^{3}$. Using these observations and assuming that $F$ is linear as a first approximation, result in $a_{\Lambda \tilde{x} q s} \approx 0.3 a_{u \tilde{c}}$ and $\phi_{\Lambda \tilde{x} q s}-\phi_{u \tilde{c}} \approx \pi$. Both the amplitude (Fig. 4a) and the phase (Fig. 4b) of the $\left\langle\Lambda_{x}\right\rangle$ modulation agrees roughly with this estimation in the quasi-steady low frequency regime. The streamwise integral scale lags the imposed unsteadiness by $\Delta t_{\tilde{\Lambda} x}^{+}=-300$ until $f^{+} \approx 0.002$, but the phase shift decreases rapidly to zero in the high frequency regime (Fig. 4b). Once more, large time lags imply large inertia as expected for the large-scale structures. But, large $\Delta t_{\tilde{\Lambda} x}^{+}$should be accompanied by steep decrease of the modulation amplitudes in simple linear relaxation systems, which is not the case for the streamwise integral scale, whose response to imposed unsteadiness is clearly non-linear.

\subsection{Taylor scale}

The Taylor hypothesis in steady flow relates the temporal to spatial derivatives according to $\partial / \partial t=-\bar{U}_{i}\left(\partial / \partial x_{i}\right)$ where $\bar{U}_{i}$ is the mean local velocity in the $i$ direction. In unsteady flows the equivalent relationship is obtained by replacing the long time average by the phase average such as $\partial / \partial t=-<U_{i}>\left(\partial / \partial x_{i}\right)$.

The temporal Taylor scale is defined by

$$
\left\langle\lambda_{T}^{2}\right\rangle=\frac{\left\langle u^{\prime} u^{\prime}\right\rangle}{\left\langle\left(\partial u^{\prime} / \partial t\right)^{2}\right\rangle} .
$$

It is related to the longitudinal Taylor length scale $\left\langle\lambda_{T x}\right\rangle$ by:

$\left\langle\lambda_{T x}\right\rangle=\sqrt{\frac{\left\langle u^{\prime} u^{\prime}\right\rangle}{\left\langle\left(\frac{\partial u^{\prime}}{\partial x}\right)^{2}\right\rangle}}=\left\langle U_{\text {con }}\right\rangle\left\langle\lambda_{T}\right\rangle$

where $\left\langle U_{\text {con }}\right\rangle$ is the phase average of the local convection velocity. The characteristics of the modulation of the Taylor scales are determined by making use of the osculating parabolas corresponding either to the phase averaged autocorrelations $\left\langle u^{\prime}\left(t^{+}, \Delta x^{+}=0\right) u^{\prime}\right.$ $\left.\left(t^{+}+\Delta t^{+}, \Delta x^{+}=0\right)\right\rangle$ or to the space-time correlations $\left\langle R_{u^{\prime} u^{\prime}}\right\rangle\left(\Delta x^{+}=10\right)=\left\langle u^{\prime}\left(t^{+}, x^{+}\right) u^{\prime}\left(t^{+}, x^{+}+\Delta x^{+}\right)\right\rangle \quad$ with zero time delay at $y^{+}=12$. 
Fig. 4 Modulation characteristics of the longitudinal integral scale at $y^{+}=12$ versus the imposed frequency. a Amplitude. b Phase
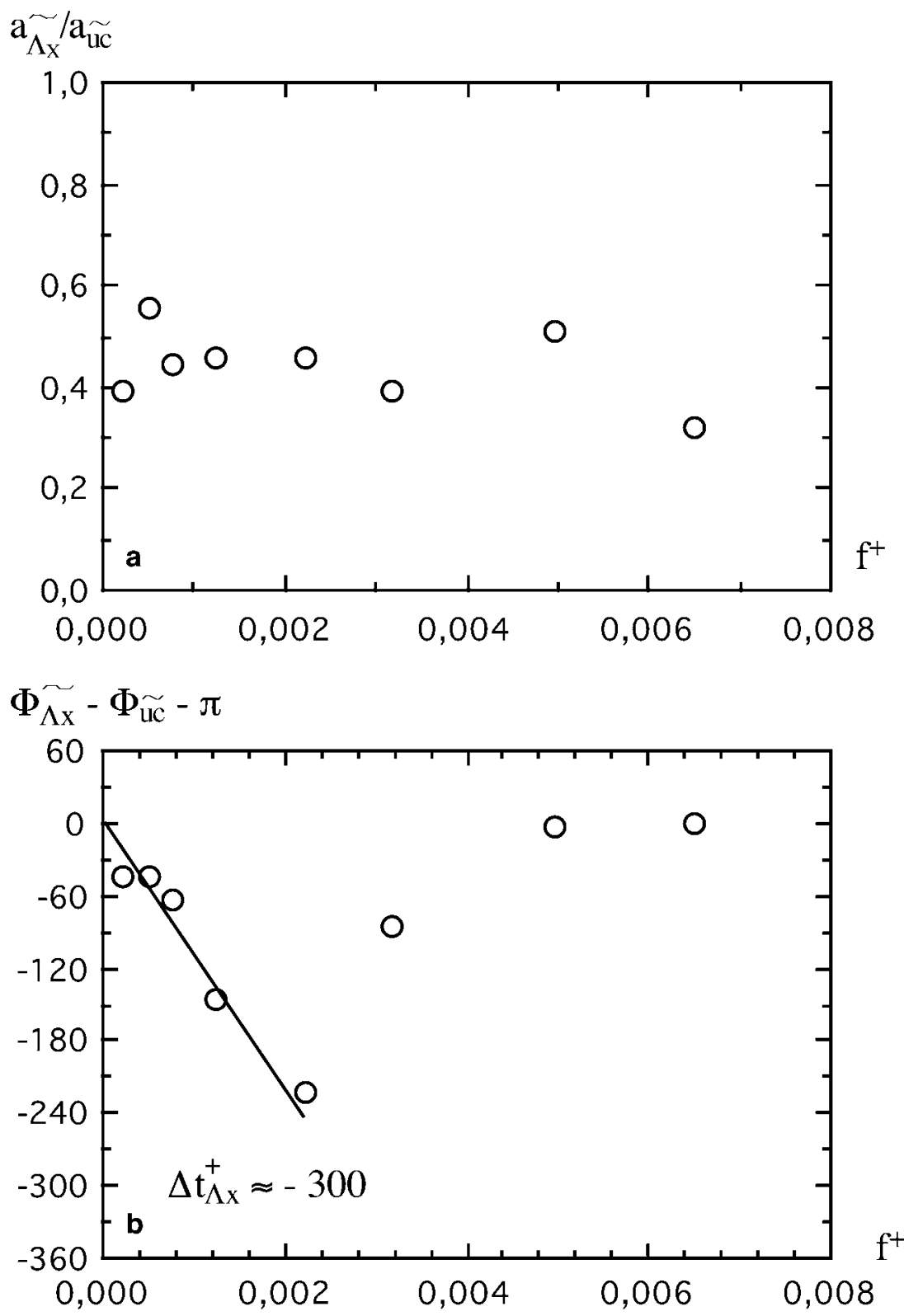

The time mean Taylor length scale $\bar{\lambda}_{T x}^{+}$is shown in Fig. 5 versus the imposed frequency. The "computed" values in this figure refer to $\bar{\lambda}_{T x}^{+}=\bar{U}_{y+=12}^{+} \bar{\lambda}_{T}^{+}$i.e. they are determined by measuring $\bar{\lambda}_{T}^{+}$and by supposing that the Taylor hypothesis is valid at the mean, with the convection velocity equal to the local time mean velocity. The measured values are those inferred from the streamwise correlation measurements. It is seen in Fig. 5 that the Taylor hypothesis is roughly well established at the mean in the range of imposed frequencies investigated here. The measured time mean Taylor length scale at $y^{+}=12$ is $\bar{\lambda}_{T x}^{+}=73$ and agrees well with Sreenivasan et al. (1983) and Direct Numerical Simulations of Antonia et al. (1991) in steady flow and Tardu et al. (1994) in unsteady flow. There is some scatter in Fig. 5 inherent to the experimental difficulties, yet the estimated Taylor scale is about $15 \%$ smaller than the measured one. The convection velocity is slightly larger than the local one with $\bar{U}_{\text {con }}^{+}=10.3$ to be compared with $\bar{U}^{+}=9$. This is in good agreement with Kim and Hussain (1992). According to these authors, the convection velocity is constant at $y^{+}<14$ and $\bar{U}_{\text {con }}^{+} \approx \bar{U}^{+}$ immediately after.

The relative amplitude and phase of $\left\langle\lambda_{T}\right\rangle$ are compared with the modulation characteristics of the wall shear stress in Fig. 6. The data of Tardu et al. (1994) are also shown in this figure for comparison. These authors determined the temporal Taylor scale in a quite different manner by computing directly $\partial u^{\prime} / \partial t$ through temporal digital filtering. They have also compared their results with the amplitude and phase of the Liepmann timescale modulation $\langle\Gamma\rangle=1 /\left(2 \pi\left\langle N_{0}\right\rangle\right)$ where $\left\langle N_{0}\right\rangle$ is the phase average of the zero-crossing frequency and have shown that it corresponds reasonably well to $\left\langle\lambda_{T}\right\rangle$. The 
Fig. 5 Time mean longitudinal Taylor length scale versus the imposed frequency at $y^{+}=12$

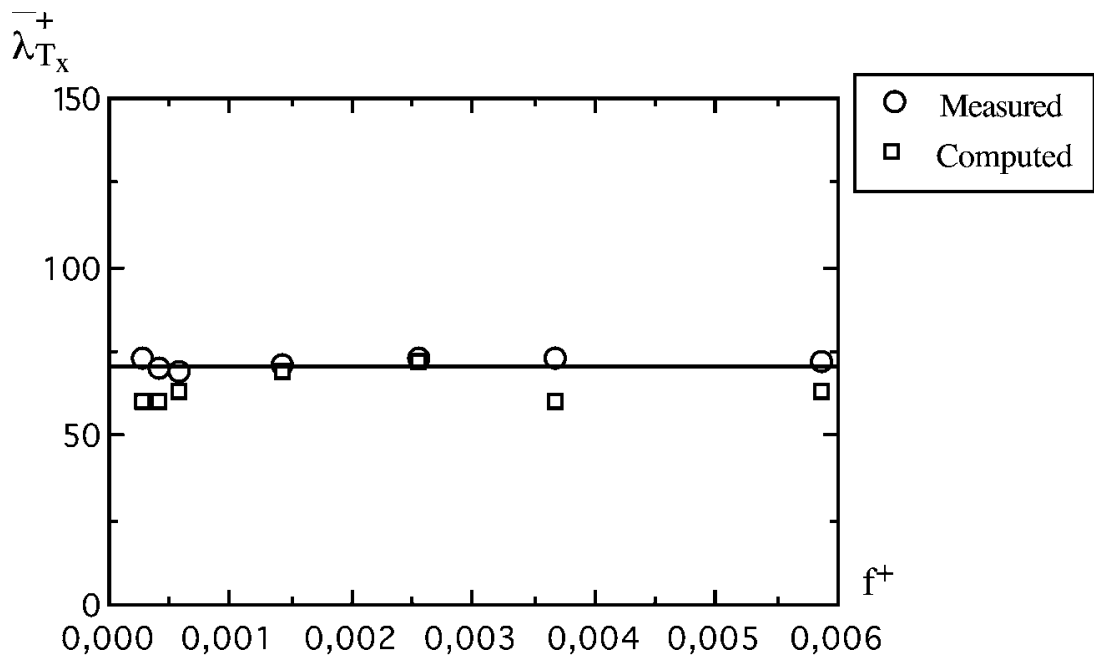

results reported here and obtained in a different manner are in good agreement with their measurements. Three interesting features of the Taylor scale's behavior have to be noted here. First, it may be shown by simple arguments that in the quasi-steady limit $a_{\tilde{\lambda} T q s} \approx a_{\tilde{\tau}}$ and $\phi_{\tilde{\lambda} T q s}-\phi_{\tilde{\tau}} \approx 180$ because $\bar{\lambda}_{T}$ scales with inner variables in steady flow. This is well established in the low imposed frequency range in Fig. 6. The amplitude $a_{\tilde{\lambda} T}$ decreases sharply at $f^{+}=0.0025$ due to the limited response time of the near wall turbulence which cannot follow the rapid modification of the imposed oscillating pressure gradient. The subsequent constant ratios $a_{\tilde{\lambda} T} / a_{\tilde{\tau}}$ indicate the beginning of a third imposed frequency regime. Similar behaviors have been noted for the wall shear stress intensity (Finnicum and Hanratty 1998; Tardu and Binder 1993), but at higher imposed frequencies.

The relative amplitude of the spatial Taylor scale $\left\langle\lambda_{T x}\right\rangle$ is related to the relative amplitude of the friction velocity $a_{\tilde{u} \tau} \approx 1 / 2 a_{\tilde{\tau}}$ in Fig. 7a. In the quasi-steady limit $a_{\tilde{\lambda} T x} \approx 3 / 4 a_{\tilde{u} \tau}$ as suggested by Tardu et al. (1994). This is followed by an increase and a decrease towards higher imposed frequencies showing that the imposed unsteadiness penetrates further into the dissipation mechanisms. The phase shift in the quasi-steady limit is $\phi_{\tilde{\lambda} T x}-\phi_{\tilde{\tau}} \approx \pi$. A time lag of about 200 wall units represents well the reaction of the Taylor length scale in the whole-imposed frequency range (Fig. 7b).

Finally Fig. 8 compares the amplitude and phase of the convection velocity $\left\langle U_{\text {con }}\right\rangle=\left\langle\lambda_{T x}\right\rangle /\left\langle\lambda_{T}\right\rangle$ with the modulation characteristics of the local velocity. The ratio $a_{\tilde{U} \text { con }} / a_{\tilde{U}}$ is about 0.9 and the phase shift $\phi_{\tilde{U} c o n}-\phi_{\tilde{U}}$ is small but systematically positive. Thus, the Taylor hypothesis in unsteady wall flows is certainly not exact in the low buffer layer, but still constitutes an acceptable approximation. We obtained closer correspondence between $\left\langle U_{\text {con }}\right\rangle$ and $\langle U\rangle$ at $y^{+}=40$ and $y^{+}=130$ (Fig.9). This is of course expected, since in steady flow the validity of the Taylor hypothesis is well established in the log layer, and the oscillating shear is confined in the buffer layer at high frequencies investigated here.
Fig. 6 Relative amplitude of the modulation of the Taylor time scale at $y^{+}=12$ compared with the modulation of the Liepmann scale and Tardu et al. (1994, triangles)

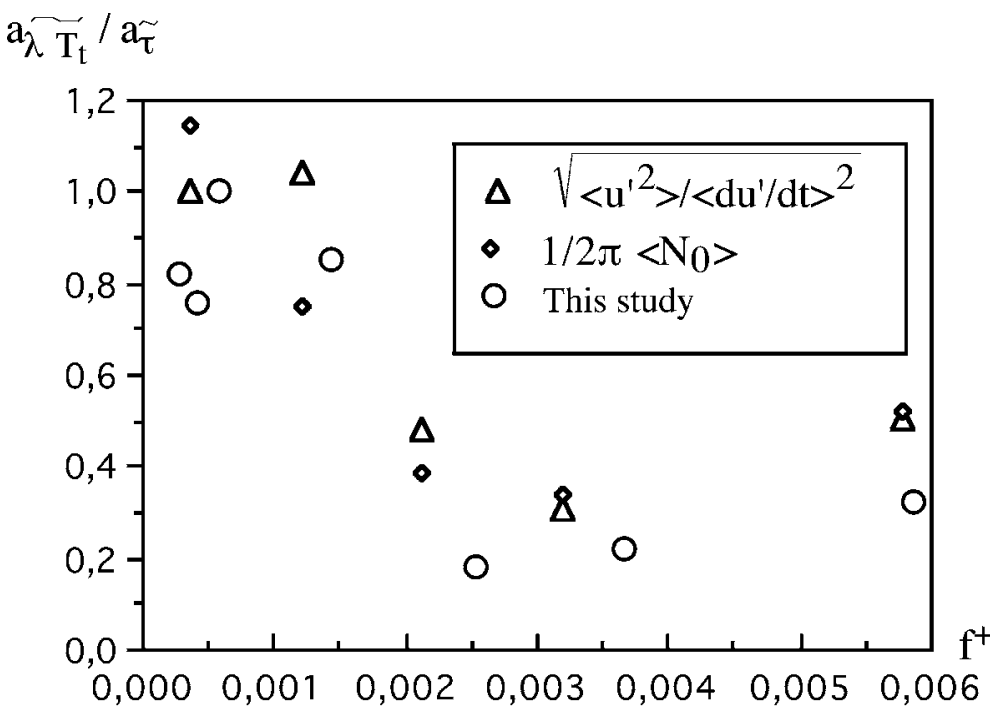


Fig. 7 Modulation characteristics (amplitude a and phase b) of the streamwise

Taylor length scale at $y^{+}=12$

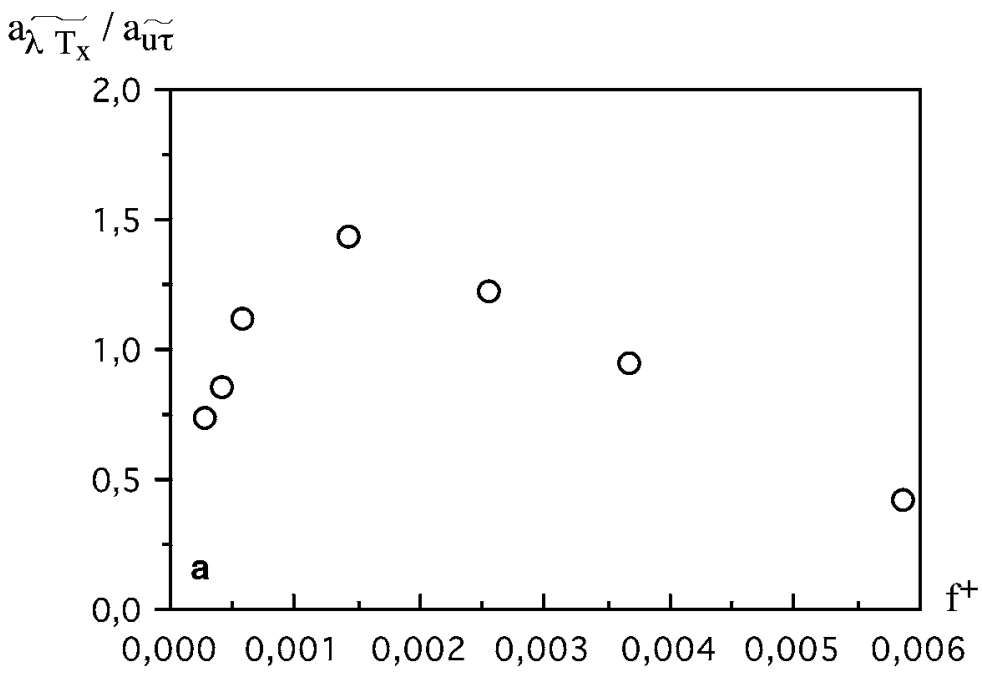

$\Phi \widetilde{\lambda T_{\mathrm{X}}}-\Phi_{\tau}^{\sim}-\pi$

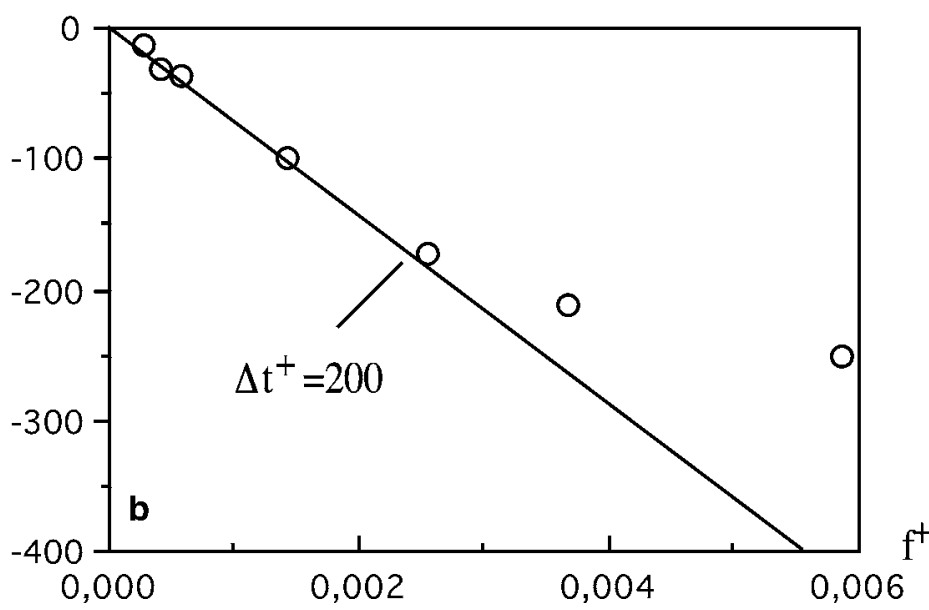

\section{Discussion}

The equations governing the time-space development of fluctuating velocity components $u_{i}$ are easily obtained by subtracting the time mean (or phase averaged) values from the equation of motion $\bar{U}_{i}+u_{i}\left(\left\langle U_{i}\right\rangle+u_{i}\right.$ in unsteady flow) and reads for (Hinze 1975 , p. 323):

$$
\begin{gathered}
\frac{\partial u_{i}}{\partial t}+u_{k} \frac{\partial \overline{U_{i}}}{\partial x_{k}}+\bar{U}_{k} \frac{\partial u_{i}}{\partial x_{k}}+\frac{\partial}{\partial x_{k}}\left(u_{i} u_{k}-\overline{u_{i} u_{k}}\right) \\
=-\frac{\partial p}{\partial x_{1}}+\frac{\partial^{2} u_{i}}{\partial x_{l} \partial x_{l}}
\end{gathered}
$$

$$
\begin{aligned}
& \frac{\partial u_{i}}{\partial t}+u_{k} \frac{\partial\left\langle U_{i}\right\rangle}{\partial x_{k}}+\left\langle U_{k}\right\rangle \frac{\partial u_{i}}{\partial x_{k}}+\frac{\partial}{\partial x_{k}}\left(u_{i} u_{k}-\left\langle u_{i} u_{k}\right\rangle\right) \\
& =-\frac{\partial p}{\partial x_{1}}+\frac{\partial^{2} u_{i}}{\partial x_{l} \partial x_{l}}
\end{aligned}
$$

For time mean unidirectional flow in the $i=1$ direction, the Taylor hypothesis implies that the following terms occurring in the instantaneous $u_{i}$ transport equation:

$$
-\frac{\partial u_{i}}{\partial x_{j}} u_{j}-\frac{\partial \bar{U}_{1}}{\partial x_{j}} u_{j}-\overline{u_{j} \frac{\partial u_{i}}{\partial x_{j}}}-\frac{1}{\rho} \frac{\partial p}{\partial x_{i}}+\frac{\partial^{2} u_{i}}{\partial x_{j} \partial x_{j}}
$$

are negligible. The Einstein summation convention applies in the last relationship and $p$ remains for the fluctuating pressure. The capital letters stay for mean or phase averaged velocities. Mixed notations are used hereafter i.e. $x_{i}$ and $u_{i}$ correspond to the streamwise, wall normal and spanwise directions $x, y, z$ and to $u^{\prime}, v^{\prime} w^{\prime}$ for respectively $i=1,2$ and 3 . All quantities are scaled with 
Fig. 8 Amplitude (a) and phase (b) of the convection velocity modulations versus the imposed frequency at $y^{+}=12$

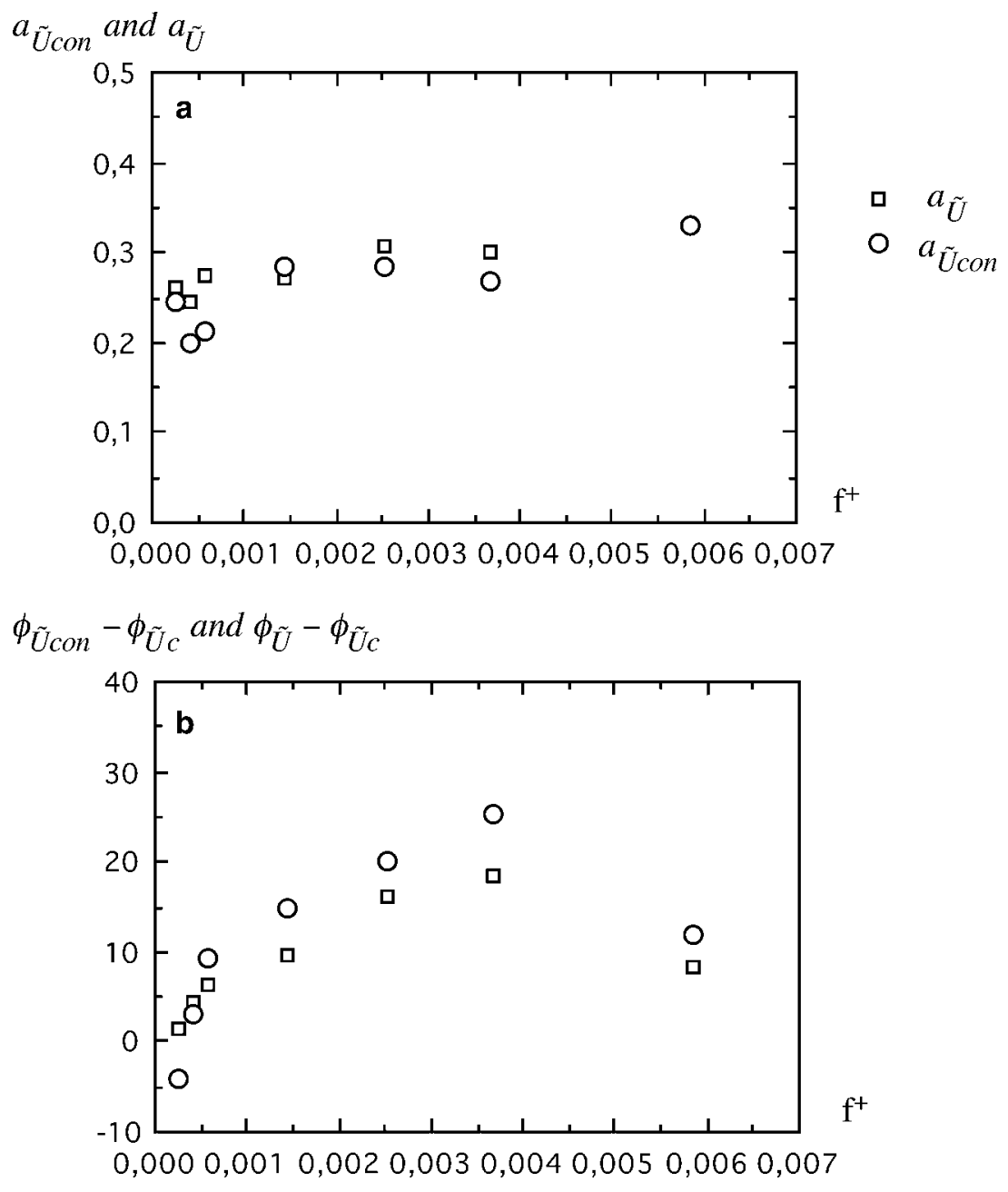

inner variables and the symbol + is omitted for the sake of simplicity.

The streamwise fluctuating velocity equation is rewritten as:

$$
\begin{aligned}
\frac{\partial u_{1}}{\partial t}= & -\bar{U} \frac{\partial u_{1}}{\partial x_{1}}-u_{2} \frac{\partial \bar{U}}{\partial x_{2}}-\frac{\partial p}{\partial x_{1}}+\frac{\partial \bar{u}_{1} u_{2}}{\partial x_{2}} \\
& +\left(-u_{1} \frac{\partial u_{1}}{\partial x_{1}}--u_{2} \frac{\partial u_{1}}{\partial x_{2}}--u_{3} \frac{\partial u_{1}}{\partial x_{3}}\right)+\frac{\partial^{2} u_{1}}{\partial x_{i} \partial x_{i}}
\end{aligned}
$$

to be consistent with the shear stress transport mechanism philosophy. The grouped terms at the right represent, respectively, the advection $A_{11}$, production $P_{11}$, the pressure $\phi_{11}$, the non-linearity $N_{11}$, the turbulent diffusion $T_{t 11}$ and finally the viscous diffusion $D_{11}$. Note that the terminology used here is in concordance with the classical definitions. The production term in the $\partial \overline{u_{1} u_{1}} / \partial t$ transport equation for example is $-2 \overline{A_{11} u_{1}}=$ $-2 \overline{u_{1} u_{2}}\left(\partial \bar{U} / \partial x_{2}\right)$ and results from multiplying the previous equation by $u_{1}$ and averaging.

It is difficult to find an easy way to check the validity of the Taylor hypothesis even if we can have access to the ensemble of these terms. The long time average of both left and right hand sides of the last equation are zero but not their absolute values. The comparison of the absolute values of the transport terms is quite convenient to check the Taylor hypothesis. We computed each term $Q$ of the $u^{\prime}$ transport equation through Direct Numerical Simulation code of Orlandi (2001) in a fully developed turbulent channel flow with $\operatorname{Re}_{\tau}=180$ using $256 \times 128 \times 128$ grid points. We determined subsequently the mean absolute values of $\overline{\left|P_{11}\right|}, \overline{\left|P_{11}+\phi_{11}\right|}, \overline{\left|P_{11}+\phi_{11}+A_{11}\right|}$ etc. by progressively adding one different term at each step and comparing them with $\left|\frac{\partial u_{1}}{\partial t}\right|$. The mean of absolute value of a random quantity is related to its rms value, as for example for a normal variable $q^{\prime}$ for which $\overline{\left|q^{\prime}\right|}=\sqrt{2 / \pi} \sqrt{\overline{q^{\prime} q^{\prime}}}$. The subsequent addition of different terms into || allows also taking account of their joint probability densities. The results are shown in Fig. 10. The following comments can be made in the light of the distributions reported in Fig. 10:

- The effects of the non-linearity and of the turbulent diffusion are negligible compared with the production, pressure and advection terms in the whole layer.

- The contribution of the viscous diffusion is significant only at $y^{+} \leq 10$. The ratio of the viscous terms to the 
Fig. 9 Comparison of the convection and local velocity modulation characteristics in the inner layer. a Amplitude, b phase
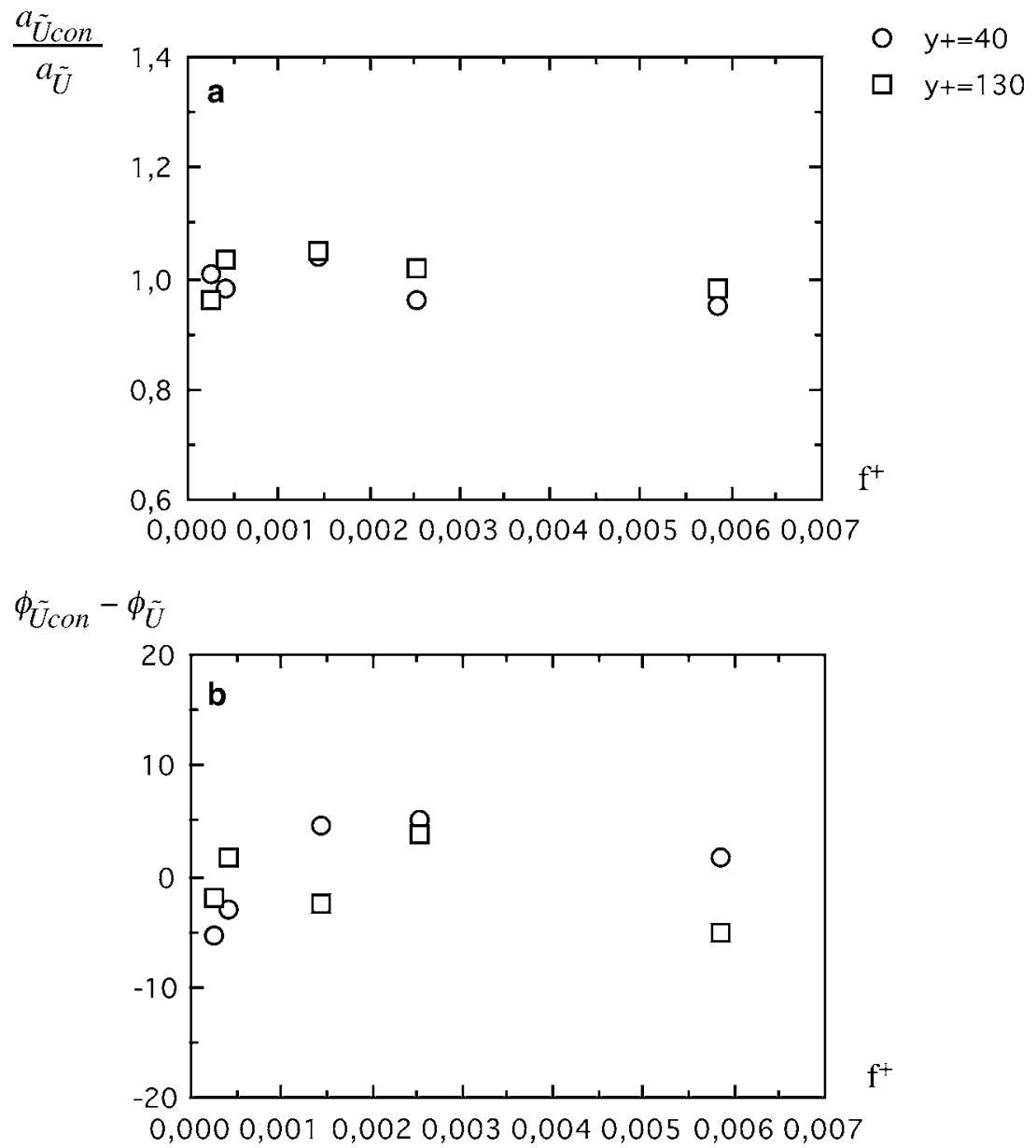

inertial ones is of the order of $\frac{1}{R e_{\lambda T x}}=\frac{v}{\sqrt{u^{\prime} u^{\prime}}} \lambda_{T x}^{-1}$ which becomes important in the viscous sublayer.

- The production and pressure gradient term $\overline{\left|P_{11}+\phi_{11}\right|}$ is dominant in the viscous sublayer but the ratio $\frac{\overline{\left|\frac{P 11}{11}+\phi_{11}\right|}}{\left|\frac{\partial u_{t}}{\partial t}\right|}$ decreases rapidly in the high buffer and log layers. It is about $1 / 3$ at $y^{+}=10$, and $1 / 8$ at $y^{+}=30$.

- The advection $A_{11}$ rapidly dominates the phenomena at $y^{+}>10$. Yet, the hypothesis of frozen turbulence is strictly valid only above the buffer layer $y^{+}>30$. It is false in the viscous sublayer and is only a crude approximation at $10<y^{+}<30$. Neglecting $\overline{\left|P_{11}+\phi_{11}\right|}$ at $y^{+}=15$ would underestimate the spatial Taylor scale by about $25 \%$ which is in rough agreement with the data of Fig. 5. This also explains the discrepancies between the modulation characteristics of the convection and local velocities in the low buffer layer in unsteady flows. The expression of the modulation characteristics through the relative amplitudes such as $a_{\tilde{U} \text { con }}=A_{\tilde{U} \text { con }} / \bar{U}_{\text {con }}$ compensates the errors made in
Fig. 10 Contribution of the transport terms to the mean absolute time-derivative of fluctuating streamwise velocity. See the text for details

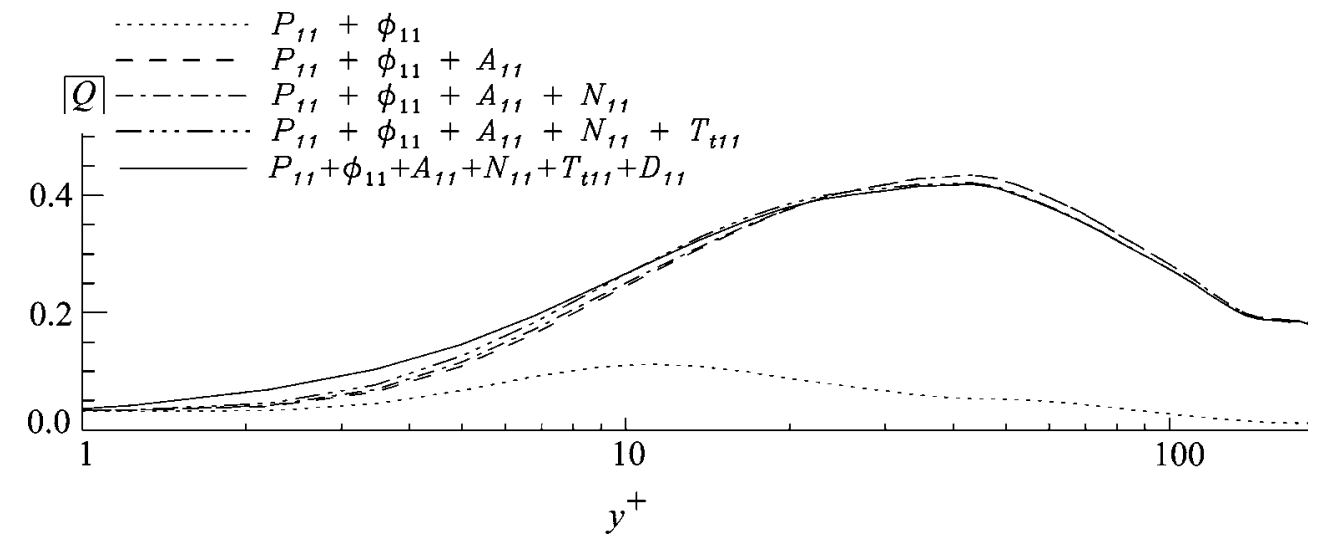


$A_{\tilde{U} \text { con }}$ and $\bar{U}_{\text {con }}$ in such a way that $a_{\tilde{U} \text { con }} \approx a_{\tilde{U}}$ at $y^{+}=12$ (Fig. 8a).

We could not experimentally investigate the wall normal and spanwise Taylor scales in this study. We have however conducted through DNS in steady flow a similar analysis for $v^{\prime}$ and $w^{\prime}$ that curiously leads to the same results. We show in Fig. 11 the transport equation results for the wall normal velocity fluctuations as an example. The transport terms are respectively $A_{22}=-\bar{U} \frac{\partial u_{2}}{\partial x_{1}}, P_{22}=0, N_{22}=\frac{\partial \overline{u_{2} u_{2}}}{\partial x_{2}}, \phi_{22}=-\frac{\partial p}{\partial x_{2}}, T_{t 22}=$ $-\left(u_{1} \frac{\partial u_{2}}{\partial x_{1}}+u_{2} \frac{\partial u_{2}}{\partial x_{2}}+u_{3} \frac{\partial u_{2}}{\partial x_{3}}\right)$ and $D_{22}=\partial^{2} u_{2} / \partial x_{i} \partial x_{i}$. It is interesting to note that the production term $P_{22}=0$ in this case, but the pressure fluctuations $\phi_{22}=-\frac{\partial p}{\partial x_{2}}$ still dominates the dynamics in the low buffer layer. At $y^{+}>30$ the major contribution to $\left|\frac{\partial v^{\prime}}{\partial t}\right|$ is from the advection term.

The validity of the Taylor hypothesis above the viscous layer is due to either smaller rms of the quantities entering in the instantaneous transport equations compared with the advection term, and/or to a specific phase relationships between them. To illustrate the latter let us rewrite the "instantaneous" equations in the form $\left(\partial u_{i} / \partial t\right)-A_{i}=\beta_{i}$ where $A_{i}$ is the advection (Taylor) term and $\beta_{i}$ are $n$ terms completing the transport equation. Suppose further for simplicity that $\beta_{i}$ are identically distributed normal variables with standard deviations $\sigma_{i}=\sigma$ and correlation coefficient $r_{i j}$, although this hypothesis is certainly false in the inner layer. Under these circumstances it can be rigorously shown that:

$\overline{\left|\frac{\partial u_{i}}{\partial t}-A_{i}\right|}=\sqrt{\frac{2}{\pi}} \sigma\left\{n+\sum_{i=1}^{n} \sum_{j=1}^{n} r_{i j}\right\}$

This relation shows clearly that $\overline{\left(\partial u_{i} / \partial t\right)-A_{i}}$ may be increasingly small either when the standard variation is small, and/or with a specific organization of the correlation coefficients in such a way that $n+\sum_{i=1}^{n} \sum_{j=1}^{n} r_{i j} \approx 0$. Piomelli et al. (1989) have investigated both the rms and correlation coefficients between $\partial u_{i} / \partial t$ and $A_{i}$. The rms values are almost identical and the correlation coefficient is larger than 0.9 above the buffer layer according to these authors. Our results are similar. The difference in the representation here is that we investigate the expected values of the absolute quantities and take into account implicitly the cross correlation between the fluctuating quantities.

Contrarily to the large eddies, small scale structures enhance the Taylor hypothesis. This has been noticed before (Sternberg 1967), and we will follow here a different reasoning to attempt to strengthen this argument. The Biot-Savart relationship gives for the fluctuating velocity field in vectorial form as an integral in a volume $V$ :

$\vec{u}(\vec{x}, t)=-\frac{1}{4 \pi} \int_{V} \frac{\left(\vec{x}-\overrightarrow{x^{\prime}}\right) \wedge \vec{\omega}\left(\overrightarrow{x^{\prime}}, t\right)}{\left\|\vec{x}-\overrightarrow{x^{\prime}}\right\|^{3}} \mathrm{~d} V^{\prime}$

in terms of the fluctuating vorticity field $\vec{\omega}\left(\overrightarrow{x^{\prime}}, t\right)$ and where || || stands for the vectorial norm. The image vorticity field is omitted in this relationship for the sake of simplicity, and that does not affect the discussion conducted here. The vorticity transport equation is given by:

$\frac{D \vec{\omega}}{D t}=\vec{\omega} \bullet \vec{\nabla} \vec{u}+v \nabla^{2} \vec{u}$

in an incompressible flow, with respectively the production and viscous terms at the right. The vorticity field is convected by the local velocity if $\overline{u_{i} u_{i}}<<\bar{U}^{2}$ and if the vorticity generation and diffusion can both be neglected. The first condition implies $y^{+} \geq 15$. The vorticity generation takes place over comparatively short times compared with the lifetime of the structures. The viscous effects can be neglected if the local $\operatorname{Re} \lambda_{T}$ is sufficiently large. The Taylor hypothesis applies for the vorticity field under these circumstances leading to $\partial \vec{\omega} / \partial x=$ $-(1 / \bar{U})(\partial \vec{\omega} / \partial t)$. Defining a local convection velocity $\bar{U}_{\text {con }}(y)$ for the streamwise fluctuating velocity component

$\frac{\partial u^{\prime}}{\partial x}=-\frac{1}{\bar{U}_{\operatorname{con}}(y)} \frac{\partial u^{\prime}}{\partial t}$,

substituting into the Biot-Savart equation and simplifying gives:
Fig. 11 Contribution of the transport terms to the mean absolute time-derivative of fluctuating wall normal velocity. Same legend as in Fig. 10. See the text for details

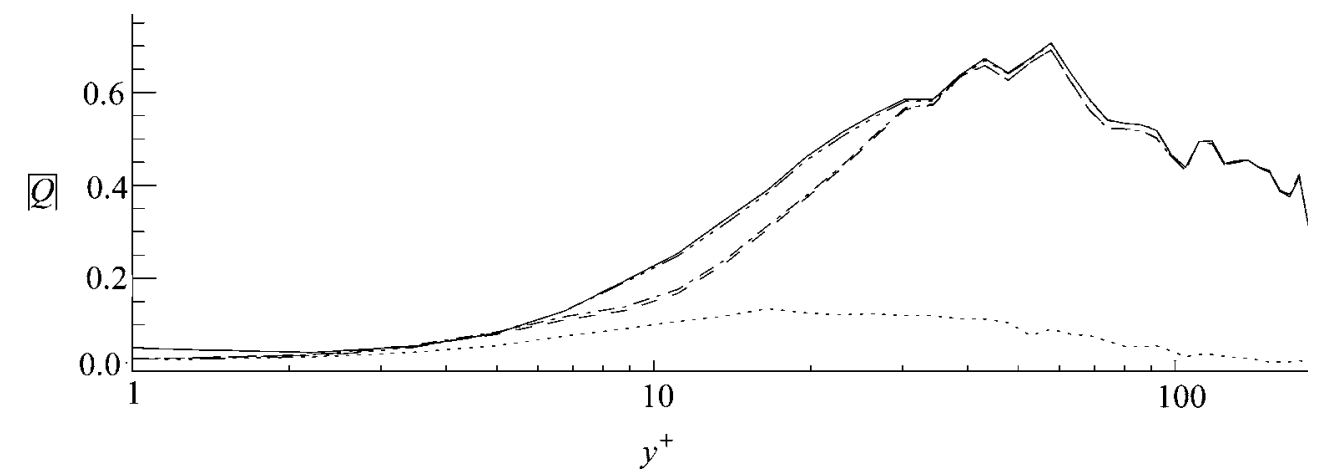




$$
\frac{1}{\bar{U}_{\text {con }}}=\frac{\int_{V} \frac{1}{\bar{U}} G_{x}\left(\vec{x}, \overrightarrow{x^{\prime}}, t\right) \mathrm{d} V^{\prime}}{\int_{V} G_{x}\left(\vec{x}, \overrightarrow{x^{\prime}}, t\right) \mathrm{d} V^{\prime}}
$$

where $G_{x}$ is the streamwise component of the vector field:

$\vec{G}=\frac{\left(\vec{x}-\overrightarrow{x^{\prime}}\right) \wedge \frac{\partial \vec{\omega}\left(\overrightarrow{x^{\prime}, t}\right)}{\partial t}}{\left\|\vec{x}-\overrightarrow{x^{\prime}}\right\|^{3}}$.

This equation shows that the hypothesis of frozen vorticity does not imply directly frozen turbulence. In the limit of infinitely well-localized vorticity of the type $G_{x}\left(\vec{x}, x^{\prime}, t\right)=\delta\left(\vec{x}-\overrightarrow{x^{\prime}}\right) H\left(\vec{x}, \overrightarrow{x^{\prime}}, t\right)$ where $\delta$ is the Dirac function one has $\bar{U}_{\text {con }}=\bar{U}$. In other words, small-scale localized vortical structures near a point $P$ strengthen the Taylor hypothesis, while the large scale eddies deviate the turbulence structure from a frozen state. This is schematically shown in Fig. 12. Near the wall the dominant structures are quasi-streamwise vortices of typical scales $s^{+} \approx 20$. The localized vorticity condition can, thus not be fulfilled at $y^{+} \leq 10$. Above the buffer layer the contribution to the convection velocity of the large scales becomes progressively negligible in an increasingly locally isotropic environment. The oscillating shear in unsteady wall flows does presumably not significantly modify this process.

\section{Conclusion}

Longitudinal spatial and temporal $\tau^{\prime}-u^{\prime}$ and $u^{\prime}-u^{\prime}$ velocity correlations are investigated in a forced turbulent channel flow within a large range of imposed frequency in the low buffer layer. Related length scales are studied with the emphasis put on the Taylor scale. The correlation coefficient is strongly modulated. The re-

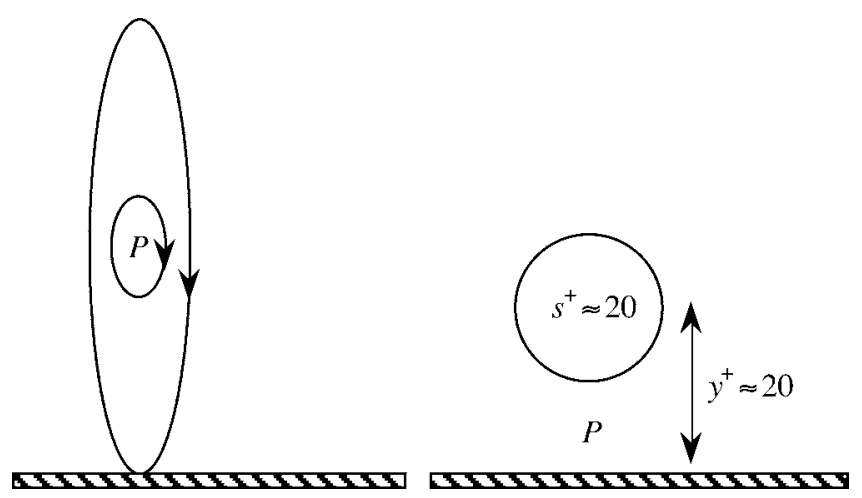

Fig. 12 Schematic view showing the effect of the large-scale and localized small-scale vortical structures on the convection velocity at a point $\mathrm{P}$ in the far buffer and log-layers (left) and near the wall in the viscous and low-buffer sublayers (right) sponse of the longitudinal integral scale to the imposed unsteadiness is astonishingly non-linear for unexplained reasons. Its amplitude is roughly constant in the whole range of $f^{+}$, but this is accompanied by important time lags which is in contradiction with the linear system responses.

The Taylor hypothesis of frozen turbulence is checked immediately above the buffer layer. It is shown that it constitutes an acceptable approximation in the presence of the oscillating shear with a good correspondence of the relative amplitudes of the convection and local velocities but small phase shifts. The mean convection velocity at $y^{+}=12$ is $15 \%$ larger than the local one. The small-scale turbulence is sensitive to the imposed unsteadiness since the amplitude and phase of the Taylor length scale vary considerably in the imposed frequency range investigated here.

To strengthen and clarify the experimental data, DNS results in a steady turbulent channel is analyzed in a way different from the previous investigations, to determine why and how the Taylor hypothesis holds remarkably valid above the low-buffer layer. The terms in the instantaneous transport equations are individually determined and their contribution to the time derivatives of the fluctuating velocity components are computed through their consecutive absolute values. It is shown that the advection rapidly dominates the phenomena at $y^{+}>10$. The deviation from the Taylor hypothesis in the low buffer and viscous sublayers is essentially due to the production and pressure gradient terms. The experimental and numerical results presented here have been obtained at relatively low Reynolds numbers, and further studies are necessary to elucidate the effect of $R e$ on the Taylor hypothesis in steady and forced wall flows.

\section{References}

Antonia RA, Kim J, Browne LWB (1991) Some characteristics of small-scale turbulence in a turbulent duct flow. J Fluid Mech 233:369

Brereton G-J, Hwang J-L (1994) The spacing of streaks in unsteady turbulent wall-bounded flow. Phys Fluids 6(7):2446

Brereton G-J, Reynolds W-C, Jarayaman R (1990) Response of a turbulent boundary layer to sinusoidal free-stream unsteadiness. J Fluid Mech 221:131

Brown GL, Thomas ASW (1977) Large structures in a turbulent boundary layer. Phys Fluids 20(10):S243

Falco RE (1977) Coherent motions in the outer region of turbulent boundary layers. Phys Fluids 20(10):S124

Finnicum D-S, Hanratty T-J (1998) Effect of imposed sinusoidal oscillations on turbulent flow in a pipe. PhysicoChem Hydrodyn 10:585

Head MR, Bandyopadhyay PR (1981) New aspects of turbulent boundary layer structure. J Fluid Mech 107:297

Kim J, Hussain F (1992) Propagation velocity and space-time correlation of perturbations in turbulent channel flow. NASA Report $\mathrm{N}^{\circ}: \mathrm{TM} 103932$

Lin CC (1953) On Taylor's hypothesis and the acceleration terms in the Navier-Stokes equation. Q Appl Math 10:295

Mao Z-X, Hanratty T-J (1986) Studies of wall shear stress in a turbulent pulsating pipe flow. J Fluid Mech 177:454 
McLean I-R (1991) The near wall eddy structure in an equilibrium turbulent boundary layer. Ph.D. Thesis, University of Southern California, USA

Orlandi P (2001) Fluid flow phenomena. Kluwer, Dordrecht

Piomelli U, Balint J-L, Wallace J-M (1989) On the validity of Taylor's hypothesis for wall-bounded flows. Phys Fluids A1(3):609

Sreenivasan KR, Prabhu A, Narasimha N (1983) Zero crossings in turbulent signals. J Fluid Mech 137:251

Sternberg J (1967) On the interpretation of space-time correlation measurements in shear flow. Phys Fluids 10(9):146-152

Tardu S, Binder G (1993) Response of turbulence to imposed oscillations of high frequencies. Phys Fluids A 5(8):2028
Tardu S, Binder G (1997) Reaction of bursting to a homogeneous oscillating pressure gradient. Eur J Mech B 16:89

Tardu S, Da Costa P (2005) Experiments and modeling of an unsteady turbulent channel flow. AIAA J 43(1):140

Tardu S, Vezin P (2004) Response of the streaks, the active and passive eddies in an unsteady channel flow. Int J Heat Fluid Flow 25:915

Tardu S, Binder G, Blackwelder R-F (1994) Turbulent channel flow with large amplitude velocity oscillations. J Fluid Mech 267:109

Tu S-W, Ramaprian B-R (1983) Fully developed periodic turbulent pipe flow. J Fluid Mech 137:31

Wark CE, Nagib HM (1991) Experimental investigation of coherent structure in turbulent boundary layer. J Fluid Mech 230:183 